\section{Sucção do recém-nascido prematuro: comparação do método Mãe-Canguru com os cuidados tradicionais}

\section{Suckling of the premature newborn child: comparison between the Kangaroo Mother method with traditional care}

Izabella Santos Nogueira de Andrade 1 Zelita Caldeira Ferreira Guedes 2

1 Universidade de Fortaleza. Av. Washington Soares, 1321. Bairro Edson Queiroz. 60811341. Fortaleza, CE, Brasil. CEP: 60.811-341. E-mail: izabellanogueira@bol.com.br. 2 Escola Paulista de Medicina. Universidade Federal de São Paulo. São Paulo, SP, Brasil

\begin{abstract}
Objectives: to compare premature newborns' suction in the Mother-Kangoroo method with newborns submitted to traditional care.

Methods: thirty premature newborns with gestational age between 30 and 35 weeks were selected. The samples were comprised of two groups: Group 1: 16 newborns of the Mother Kangoroo Ward of the Maternity School Assis Chateaubriand and Group 2: formed by 14 newborns submitted to traditional care, i.e. Medium Risk Nursery of the General Hospital of Fortaleza. All newborns were evaluated and submitted to phoniatric intervention in the process of hospital discharge.

Results: in Group 1 there was a significant improvement related to behavior, stress signs, coordination and suction rhythm. Hospital stay was considerably shorter. In group 2 there was a significant improvement related to the coordination between suction, deglutition and breathing.

Conclusions: newborns of both groups were benefited by phoniatrics, nevertheless, the best results related to the Kangaroo-Mother Method. It has been noted that this method is a good alternative for developing countries for it contributes to the effectiveness of nursing, shortening hospital stay and reducing public health costs.

Key words Kangaroo Mother Method, Infant premature, Breast feeding
\end{abstract}

\section{Resumo}

Objetivos: comparar o processo de sucção em recém-nascidos prematuros incluídos no método MãeCanguru com recém-nascidos submetidos aos cuidados tradicionais.

Métodos: foram selecionados 30 recém-nascidos prematuros com idade gestacional entre 30 e 35 semanas. A amostra foi constituida de dois grupos: Grupo 1, composto por 16 recém-nascidos inseridos no Alojamento Mãe-Canguru da Maternidade Escola Assis Chateaubriand e Grupo 2, constituido por 14 recém-nascidos submetidos aos cuidados tradicionais, ou seja, Berçário de Médio Risco do Hospital Geral de Fortaleza. Todos os recém-nascidos foram avaliados, submetidos à intervenção fonoaudiológica e reavaliados durante a alta hospitalar.

Resultados: no Grupo 1 houve uma melhora significativa em relação ao estado comportamental, sinais de estresse, coordenação e ritmo de sucção. $O$ tempo de permanência hospitalar foi consideravelmente menor. No Grupo 2 foi verificada uma melhora significativa em relação à coordenação entre sucção, deglutição e respiração.

Conclusões: os recém-nascidos de ambos os grupos foram beneficiados com a intervenção fonoaudiológica, no entanto, os melhores resultados foram referentes ao método Mãe-Canguru. Verificou-se que esse método constitui uma ótima alternativa para paises em desenvolvimento, pois contribui para a efetividade da amamentação, diminuindo o tempo de permanência hospitalar, acarretando menores custos para a saúde pública.

Palavras-chave Método Mãe-Canguru, Prematuro, Aleitamento materno. 


\section{Introdução}

A realidade socioeconômica dos países em desenvolvimento demonstra o significativo número de nascimentos de recém-nascidos prematuros, o que causa um elevado percentual de morbidade e mortalidade neonatal e, quando não, a necessidade de permanecerem em unidades de terapia intensiva neonatal (UTIN), acarretando alto custo financeiro e necessidade de aparelhagem. ${ }^{1}$

Diante dessa realidade, em 1978, Edgar Rey Sanabria e posteriormente Héctor Martínez Gómez, médicos do Instituto Médico Infantil (IMI) de Bogotá, Colômbia, elaboraram um programa denominado Mãe-Canguru. Tal nome foi adotado em referência as espécies dos marsupiais, cujos cangurus fêmeas possuem uma bolsa onde as suas crias prematuras completam o tempo de gestação, sendo aquecidas e alimentadas até se fortalecerem e amadurecerem.

A proposta do método Mãe-Canguru é que, da mesma forma que os cangurus, as mães de bebês prematuros irão carregar os seus filhos, quando esses se encontrarem em condições clínicas, gástricas e respiratórias que viabilizem uma situação estável. Charpak et al.1,2 relatam como objetivos principais do método suprir a insuficiência de recursos materiais, usando-se como alternativa a substituição de incubadoras e evitando a separação prolongada entre a mãe e seu bebê.

O Método Mãe-Canguru tem sido utilizado em diversos países, adaptando-se para cada um, adequando-se às próprias condições culturais e às necessidades locais. Devem ser enfatizados, contudo, três componentes comuns: posição canguru, nutrição baseada no aleitamento materno e alta hospitalar com acompanhamento ambulatorial. No Brasil, o método iniciou-se em uma maternidade de Santos e posteriormente em 1994, no Instituto Materno Infantil de Pernambuco. ${ }^{1}$

Atualmente, no Brasil, vem-se trabalhando com a visão de um novo paradigma, que é o de atenção humanizada à criança e à família, em função de avanços tecnológicos que têm aumentado a sobrevivência e as perspectivas de desenvolvimento de recém-nascidos prematuros. Novos procedimentos sistematizados estão sendo valorizados, influenciando a qualidade da interação mãe-bebê, a comunicação, audição, o desenvolvimento global e a adequação da alimentação. Isso justifica a inclusão do fonoaudiólogo como integrante da equipe multidisciplinar do ambiente hospitalar.

No Método Mãe-Canguru, empiricamente, partese do princípio de que a intervenção fonoaudiológica será favorecida pela díade mãe-bebê. Questiona-se, porém, até que ponto a posição canguru facilitará os aspectos referentes à comunicação e alimentação de bebês prematuros.

Os recém-nascidos prematuros, em razão da própria imaturidade cerebral, têm dificuldades de permanecer em estado de alerta, apresentando um tônus predominantemente extensor, reflexos orais ausentes ou incompletos, além de uma série de fatores que podem explicar as dificuldades de sucção e incoordenação com deglutição e respiração, retardando no ganho de peso e, conseqüentemente, prolongando a alta hospitalar. 3-7

Ciente dessas condições, o presente estudo tem como objetivo comparar o processo de sucção entre bebês prematuros inseridos no Método Mãe-Canguru e aqueles submetidos aos cuidados tradicionais.

\section{Métodos}

Este estudo foi delineado como descritivo, comparativo e prospectivo. Desenvolveu-se no período de julho a novembro de 2002 na Maternidade Escola Assis Chateaubriand (MEAC) e de novembro de 2002 a maio de 2003 no Hospital Geral de Fortaleza (HGF), Ceará, Brasil.

A população estudada foi constituída de recémnascidos prematuros com idade gestacional entre 30 e 35 semanas e seis dias (Capurro somático), de ambos os sexos, com diagnóstico médico de prematuridade, adequados para idade gestacional, podendo apresentar problemas respiratórios, o que é comum nos prematuros nascidos nos referidos hospitais.

Foram elegíveis mães que aceitaram participar da pesquisa e que estavam dispostas a receber orientações e acompanhar seus bebês até a alta hospitalar. Os critérios de seleção materna incluíram gestação sem intercorrências significativas, tais como: sangramentos durante o primeiro trimestre, pré-eclâmpsia e história de abuso de drogas. Para inserção do recémnascido na pesquisa foram considerados: idade gestacional ao nascimento de 30 a 35 semanas e seis dias, nutrição enteral através do leite materno, peso de nascimento mínimo de $1080 \mathrm{~g}$, Apgar acima de 6 no 5', além do estado comportamental adequado no momento da avaliação, ou seja, estado de alerta com inatividade, segundo a classificação de Brazelton. ${ }^{6}$

Foram considerados como critérios de exclusão bebês com seqüelas perinatais como: hipóxia severa, hemorragia intracraniana, problemas pulmonares, cardíacos, neurológicos, síndromes e malformações.

A amostra estudada foi constituída por dois grupos: o Grupo 1 foi composto de recém-nascidos inseridos no alojamento Mãe-Canguru (AMC) da 
MEAC; e o Grupo 2, de recém-nascidos prematuros internados no berçário de médio risco II (BMRII), do HGF.

No período em que foi realizada a pesquisa do Grupo 1, houve 47 recém-nascidos internos no alojamento Canguru, permanecendo 16 na pesquisa que corresponderam aos critérios de inclusão acima descritos.

No Grupo 2 foram avaliados 29 bebês, de um total de aproximadamente 98 recém-nascidos internos no BMR II, também correspondente aos critérios esperados, no entanto, apenas 14 fizeram parte da pesquisa, devido a algumas dificuldades imprevistas, como: alta hospitalar antes da reavaliação (oito bebês); não comparecimento das mães no processo de intervenção (cinco bebês) e retorno à Unidade de Terapia Intensiva Neonatal de alto risco (dois bebês).

Para efetivação da pesquisa, considerando-se os critérios e as dificuldades encontradas, foram selecionadas 30 duplas (mães e bebês): 16 do grupo em estudo (MMC) e 14 do grupo controle (BMRII).

Inicialmente foi realizada avaliação, intervenção e reavaliação fonoaudiológica no grupo em estudo (MMC). Posteriormente, os mesmos procedimentos foram aplicados no grupo controle (BMRII).

A coleta de dados foi realizada através de um protocolo de avaliação fonoaudiológica que também foi utilizado na reavaliação que ocorreu durante a alta hospitalar.

Neste protocolo foram considerados os dados relevantes de anamnese, tais como: identificação, gestação, condições de parto e complicações neonatais.

A avaliação, assim como a reavaliação, foi realizada em dois momentos. O primeiro abordou aspectos referentes à postura em repouso. $5 \mathrm{O}$ tônus foi avaliado de forma passiva e ativa. ${ }^{7}$ Os estados de consciência e sinais de estresse foram avaliados de acordo com a escala de avaliação neurocomportamental conforme padronizado por Brazelton. 6

O segundo momento enfatizou a avaliação da sucção não-nutritiva, verificada através do dedo enluvado, e na sucção nutritiva, o seio materno. ${ }^{3,8-10}$

$\mathrm{Na}$ sucção não-nutritiva (SNN) foram verificadas a presença ou ausência de sucção, a força, o ritmo, as pausas e a coordenação com deglutição. Foram analisados também o vedamento labial e a coordenação dos movimentos de mandíbula e língua. $\mathrm{Na}$ sucção nutritiva (SN) foi investigado quando o recém-nascido iniciou a alimentação por via oral, avaliando-se a postura, o tempo que permanecia sugando, a predominância do estado de consciência, além de todos os itens citados na SNN.

A intervenção fonoaudiológica ocorreu de acordo com o que foi detectado na avaliação. Foi priorizada a sucção não-nutritiva com o dedo enluvado antes do horário das mamadas e durante o complemento da alimentação via sonda orogástrica, visando o amadurecimento do reflexo de sucção e coordenação com deglutição e respiração. Durante a intervenção, o bebê deveria estar em decúbito lateral e em estado de alerta com inatividade (estado 4). 3,6,8,10

Posteriormente, durante a alta hospitalar, os bebês foram reavaliados, seguindo os mesmos critérios das avaliações iniciais. Nesse momento, porém, foram considerados o peso de alta e o tempo de permanência hospitalar.

A seleção das variáveis pertinentes ao estudo contempla os aspectos referentes à alimentação de recém-nascidos prematuros inseridos no AMC e UTIN, considerando os dados de anamnese, avaliação e reavaliação. No que se refere aos dados de anamnese foram considerados: a) idade gestacional: (de 30 a 35 semanas e seis dias); b) gênero; c) peso; d) Apgar 5': (maior ou igual a 7); e) assistência ventilatória: (ventilador mecânico, CPAP, oxihood); f) fototerapia; g) tempo de UTI; h) tempo de permanência hospitalar; i) peso de alta; j) no que diz respeito à avaliação e reavaliação foram considerados: tônus: (diminuído, aumentado e normal), reação de busca; (presença/ausência do reflexo).

Os próximos itens referem-se tanto a sucção nãonutritiva quanto à nutritiva: a) força de sucção: (forte, média e fraca); b) ritmo: (três a cinco sucções por rajada/mais de cinco sucções por rajada); c) coordenação: (presença/ausência da coordenação entre sucção, deglutição e respiração; d) pausas: (presença/ausência de pausas); e) sinais de estresse: (escapes de leite; soluço; alteração respiratória; sustos e tremores; engasgos; variação do tônus, dentre outros).

Os dados obtidos foram codificados e digitados no programa Excel XP, sendo então encaminhados para o estudo estatístico.

Inicialmente foi feita a seleção de todos os dados coletados e em seguida, por meio de análise descritiva, foi possível a detecção dos achados mais relevantes, possibilitando algumas associações. Neste momento, os dados foram processados no software SPSS (Statistical Package Social Science), versão 10.0 .

Para analisar a associação entre as variáveis escolhidas, utilizou-se: a) teste de $\chi^{2}$, quando indicado; b) teste exato de Fisher, para as Tabelas $2 \times 2 \mathrm{e}$ com freqüências muito baixas; c) cálculos de média e desvio padrão, considerando o coeficiente de variação $\geq 25 \%$ para as variáveis referentes à idade gestacional, peso, Apgar no 1' e no 5', tempo de 
assistência ventilatória, tempo de internação em UTIN, tempo de hospitalização e peso na alta hospitalar.

Adotou-se, em todos os testes, o nível de significância de $5,0 \%$.

O Comitê de Ética da Escola Paulista de Medicina da Universidade Federal de São Paulo analisou e concedeu a aprovação do projeto de pesquisa com o protocolo CEP n. ${ }^{\circ} 0491 / 03$.

Todas as mães foram previamente informadas sobre a pesquisa e após concordarem em participar, assinaram um termo de consentimento livre e esclarecido, de acordo com a Resolução n. ${ }^{\circ}$ 196/96 do Conselho Nacional de Saúde do Ministério da Saúde.

\section{Resultados}

As Tabelas 1 e 2 apresentam as intercorrências perinatais em ambos os grupos, assim como as características da sucção dos recém-nascidos prematuros, que foram identificadas através da avaliação.

Tabela 1

Dados de anamnese e intercorrências perinatais nos Grupos 1 e 2, realizados na Maternidade Escola Assis Chateaubriand e no Hospital Geral de Fortaleza. Fortaleza, Ceará, julho de 2002 a maio de 2003.

\begin{tabular}{|c|c|c|c|c|c|c|c|c|}
\hline \multirow{2}{*}{ Variáveis } & \multicolumn{4}{|c|}{$\begin{array}{c}\text { Grupo } 1 \text { (MMC) } \\
N=16\end{array}$} & \multicolumn{4}{|c|}{$\begin{array}{c}\text { Grupo } 2 \text { (BRMII) } \\
N=14\end{array}$} \\
\hline & $\bar{X} \pm d p$ & Min & Max & CV & $\bar{X} \pm d p$ & Min & Max & CV \\
\hline Idade gestacional (d) & $233 \pm 10,6$ & 210 & 248 & 4,5 & $233 \pm 14,3$ & 203 & 252 & 6,1 \\
\hline Peso (g) & $1809 \pm 221,4$ & 1335 & 2120 & 12,2 & $1702 \pm 298,6$ & 1080 & 2190 & 17,5 \\
\hline Apgar $5^{\prime}$ & $8,1 \pm 0,8$ & 7 & 9 & 9,8 & $8,4 \pm 0,64$ & 7 & 9 & 7,6 \\
\hline Assitência ventilatória & $5 \pm 3,2$ & 2 & 12 & $64,0 *$ & $8,1 \pm 3,8$ & 2 & 14 & 46,9 * \\
\hline Tempo de UTI (d) & $7,1 \pm 4,1$ & 2 & 15 & $57,7 *$ & $121,8 \pm 12,6$ & 2 & 53 & 10,3 \\
\hline Tempo de hospitalização (d) & $20 \pm 10,3$ & 9 & 45 & $51,5^{*}$ & $26 \pm 15$ & 9 & 68 & 57,6 * \\
\hline Peso de alta (g) & $1847 \pm 128,6$ & 1670 & 2080 & 6,9 & $1893 \pm 150$ & 1735 & 2176 & 7,9 \\
\hline
\end{tabular}

$\mathrm{CV}=$ coeficiente de variação; Min = mínimo; Max = máximo; ${ }^{*} \mathrm{CV}>25 \% ; \mathrm{T}$. UTI = Tempo de Unidade de Terapia Intensiva Neonatal; MMC = Método Mãe Canguru; BMRII = Berçário Médio Risco 2.

Na Tabela 1, verifica-se uma homogeneidade nos grupos estudados referentes à idade gestacional $(\mathrm{G} 1$ $=4,5$ e G2 $=6,1)$, peso de nascimento $(\mathrm{G} 1=12,2 \mathrm{e}$ $\mathrm{G} 2=17,5)$, Apgar no $5^{\prime}(\mathrm{G} 1=9,8$ e G2 $=7,6)$ e no peso de alta hospitalar $(\mathrm{G} 1=6,9$ e G2 $=7,9)$. No entanto, quando comparado o coeficiente de variação (CV) referente ao tempo de hospitalização, foi verificado que houve uma diferença superior a $25 \%$, ou seja, no G1 $=51,5$ e o $\mathrm{G} 2=57,6 \%$.

De acordo com a Tabela 2, observou-se que a maior parte das crianças foi avaliada em estado de alerta com inatividade. No que se refere aos sinais de estresse, foi verificada uma diferença estatistica- mente significativa em relação ao Grupo 1, enquanto que no Grupo 2 todos os bebês apresentaram sinais de estresse.

Verificou-se uma diferença estatisticamente significativa em relação ao estado comportamental e aos sinais de estresse no que se refere ao Grupo 1. (Tabela 3) No que tange a coordenação e as pausas, vale ressaltar que estavam presentes em todos os bebês; no entanto, a melhora ocorreu primeiro no Grupo 1 (MMC). É importante enfatizar que a reavaliação foi realizada após a intervenção fonoaudiológica, no momento da alta hospitalar. 
Ocorrência da avaliação da alimentação nos Grupos 1 e 2, realizados na Maternidade Escola Assis Chateaubriand e no Hospital Geral de Fortaleza. Fortaleza, Ceará, julho de 2002 a maio de 2003.

\begin{tabular}{|c|c|c|c|c|c|c|c|}
\hline \multicolumn{8}{|c|}{ Grupos } \\
\hline & \multicolumn{2}{|c|}{ Grupo 1 (MMC) } & \multicolumn{2}{|c|}{ Grupo 2 (BMRII) } & \multicolumn{2}{|c|}{ Total } & \multirow[t]{2}{*}{$p$} \\
\hline & $\mathrm{n}$ & $\%$ & $\mathrm{n}$ & $\%$ & $\mathrm{n}$ & $\%$ & \\
\hline \multicolumn{8}{|l|}{ Estado comportamental } \\
\hline Sonolência & 4 & 25,0 & - & - & 4 & 13,3 & \\
\hline Alerta inativo & 12 & 75,0 & 10 & 71,4 & 22 & 73,3 & 0,136 \\
\hline Alerta ativo & - & - & 4 & 28,5 & 4 & 13,3 & \\
\hline \multicolumn{8}{|l|}{ Postura } \\
\hline Extensora & 5 & 31,2 & 7 & 50,0 & 12 & 40,0 & 0,452 \\
\hline Flexão de membros inferiores & 11 & 68,7 & 7 & 50,0 & 18 & 60,0 & \\
\hline \multicolumn{8}{|l|}{ Ritmo } \\
\hline Sim & 5 & 31,2 & 5 & 35,7 & 10 & 33,3 & 1,000 \\
\hline Não & 11 & 68,8 & 9 & 64,3 & 20 & 66,7 & \\
\hline \multicolumn{8}{|l|}{ Coordenação } \\
\hline Sim & 12 & 75,0 & 8 & 57,1 & 20 & 66,7 & 0,442 \\
\hline Não & 4 & 25,0 & 6 & 42,8 & 10 & 33,3 & \\
\hline \multicolumn{8}{|l|}{ Pausas } \\
\hline Sim & 13 & 81,2 & 11 & 78,5 & 24 & 80,0 & 1,000 \\
\hline Não & 3 & 18,7 & 3 & 21,4 & 6 & 20,0 & \\
\hline \multicolumn{8}{|l|}{ Sinais de estresse } \\
\hline Ausente & 5 & 31,2 & - & - & 5 & 16,6 & 0,045 \\
\hline Presente & 11 & 68,7 & 14 & 100,0 & 25 & 83,3 & \\
\hline
\end{tabular}

MMC = Método Mãe-Canguru; BMRII = Berçário Médio Risco 2.

Dados da reavaliação da alimentação nos Grupos 1 e 2, realizados na Maternidade Escola Assis Chateaubriand e no Hospital Geral de Fortaleza. Fortaleza, Ceará, julho de 2002 a maio de 2003.

\begin{tabular}{|c|c|c|c|c|c|c|c|}
\hline \multicolumn{8}{|c|}{ Grupos } \\
\hline & \multicolumn{2}{|c|}{ Grupo 1 (MMC) } & \multicolumn{2}{|c|}{ Grupo 2 (BMRII) } & \multicolumn{2}{|c|}{ Total } & \multirow[t]{2}{*}{$p$} \\
\hline & $\mathrm{n}$ & $\%$ & $n$ & $\%$ & $\mathrm{n}$ & $\%$ & \\
\hline \multicolumn{8}{|l|}{ Estado comportamental } \\
\hline Sonolência & 1 & 6,2 & - & - & 1 & 3,3 & \\
\hline Alerta inativo & 15 & 93,7 & 9 & 64,2 & 24 & 80,0 & 0,017 \\
\hline Alerta ativo & - & - & 5 & 35,7 & 5 & 16,6 & \\
\hline \multicolumn{8}{|l|}{ Postura } \\
\hline Extensora & 1 & 6,2 & 4 & 28,6 & 5 & 16,7 & \\
\hline Flexão de membros inferiores & 11 & 68,8 & 8 & 57,1 & 19 & 63,3 & 0,157 \\
\hline $\begin{array}{l}\text { Flexão de membros superiores e } \\
\text { membros superiores }\end{array}$ & 4 & 25,0 & 2 & 14,3 & 6 & 20,0 & \\
\hline \multicolumn{8}{|l|}{ Ritmo } \\
\hline Sim & 13 & 81,2 & 9 & 64,2 & 22 & 73,3 & 0,417 \\
\hline Não & 3 & 18,8 & 5 & 35,7 & 8 & 26,7 & \\
\hline \multicolumn{8}{|l|}{ Sinais de estresse } \\
\hline Ausente & 10 & 62,5 & - & - & 10 & 33,3 & $0<001$ \\
\hline Presente & 6 & 37,5 & 14 & 100,0 & 20 & 66,6 & \\
\hline
\end{tabular}

MMC = Método Mãe-Canguru; BMRII = Berçário Médio Risco 2. 
Comparação da avaliação da alimentação com a reavaliação no Grupo 1, realizada na Maternidade Escola Assis Chateaubriand. Fortaleza, Ceará, julho a novembro de 2002.

\begin{tabular}{|c|c|c|c|c|c|}
\hline & \multicolumn{4}{|c|}{ Grupo 1 (MMC) } & \multirow{3}{*}{$p$} \\
\hline & \multicolumn{2}{|c|}{ Avaliação } & \multicolumn{2}{|c|}{ Reavaliação } & \\
\hline & $\mathrm{n}$ & $\%$ & $\mathrm{n}$ & $\%$ & \\
\hline \multicolumn{6}{|c|}{ Estado comportamental } \\
\hline Sonolência & 4 & 25,0 & 1 & 6,2 & \\
\hline Alerta inativo & 12 & 75,0 & 15 & 93,7 & 0,333 \\
\hline \multicolumn{6}{|l|}{ Postura } \\
\hline Extensora & 5 & 31,2 & 1 & 6,2 & \\
\hline Flexão MMII & 11 & 68,7 & 11 & 68,7 & 0,196 \\
\hline Flexão & - & - & 4 & 25,0 & \\
\hline \multicolumn{6}{|l|}{ Ritmo } \\
\hline Presente & 5 & 31,2 & 13 & 81,2 & 0,011 \\
\hline Ausente & 11 & 68,8 & 3 & 18,7 & \\
\hline \multicolumn{6}{|l|}{ Coordenação } \\
\hline Presente & 12 & 75,0 & 16 & 100,0 & 0,101 \\
\hline Ausente & 4 & 25,0 & - & - & \\
\hline \multicolumn{6}{|l|}{ Pausas } \\
\hline Presente & 13 & 81,2 & 16 & 100,0 & 0,101 \\
\hline Ausente & 3 & 18,7 & - & - & \\
\hline \multicolumn{6}{|l|}{ Sinais de estresse } \\
\hline Ausente & 5 & 31,2 & 10 & 62,5 & \\
\hline Presente & 11 & 68,7 & 6 & 37,5 & 0,156 \\
\hline
\end{tabular}

Tabela 5

Comparação da avaliação da alimentação com a reavaliação no Grupo 2, no Hospital Geral de Fortaleza. Fortaleza, Ceará, no período de novembro de 2002 a maio de 2003.

\begin{tabular}{|c|c|c|c|c|c|}
\hline & \multicolumn{4}{|c|}{ Grupo 2 (BRMII) } & \multirow{3}{*}{$p$} \\
\hline & \multicolumn{2}{|c|}{ Avaliação } & \multicolumn{2}{|c|}{ Reavaliação } & \\
\hline & $\mathrm{n}$ & $\%$ & $\mathrm{n}$ & $\%$ & \\
\hline \multicolumn{6}{|l|}{ Estado comportamental } \\
\hline Alerta inativo & 10 & 71,4 & 9 & 64,2 & \\
\hline Alerta ativo & 4 & 28,5 & 5 & 35,7 & 1,000 \\
\hline \multicolumn{6}{|l|}{ Postura } \\
\hline Extensora & 7 & 50,0 & 4 & 28,6 & 0,453 \\
\hline Flexão membros inferiores & 7 & 50,0 & 8 & 57,1 & \\
\hline Flexão & - & - & 2 & 14,3 & \\
\hline \multicolumn{6}{|l|}{ Ritmo } \\
\hline Presente & 5 & 35,7 & 9 & 64,2 & 0,257 \\
\hline Ausente & 9 & 64,2 & 5 & 35,7 & \\
\hline \multicolumn{6}{|l|}{ Coordenação } \\
\hline Presente & 8 & 57,1 & 14 & 100,0 & 0,016 \\
\hline Ausente & 6 & 42,8 & - & - & \\
\hline \multicolumn{6}{|l|}{ Pausas } \\
\hline Presente & 11 & 78,5 & 14 & 100,0 & 0,222 \\
\hline Ausente & 3 & 21,4 & - & - & \\
\hline
\end{tabular}

BMRII = Berçário Médio Risco 2. 
As Tabelas 4 e 5 correspondem à comparação entre a avaliação e reavaliação em ambos os grupos.

Conforme a Tabela 4 foi verificada uma diferença significante em relação ao ritmo de sucção no grupo MMC.

Quanto ao grupo BRMII verificou-se aumento da coordenação (sucção, deglutição e respiração) na reavaliação $(p<0,016)$.

\section{Discussão}

De acordo com os dados de anamnese e intercorrências perinatais nos Grupos 1 (MMC) e 2 (BMR II) verificou-se que a média de idade gestacional da amostra da pesquisa foi de 33 semanas, o que caracteriza essa amostra como prematuros moderados. Em relação ao peso, foi observado que a média do Grupo 1 foi de $1809 \mathrm{~g}$, enquanto que no Grupo 2 foi de $1702 \mathrm{~g}$, sendo portanto considerados recém-nascidos de baixo peso.12-14

A maior parte da amostra foi constituída de recém-nascidos prematuros moderados e de baixo peso, o que facilitou a análise da pesquisa, apesar de a escolha ter sido aleatória, considerou-se os critérios de inclusão e exclusão, pois recém-nascidos prematuros extremos ou de muito baixo peso apresentariam um maior número de intercorrências, maiores chances de retornarem a UTIN de alto risco e, portanto, serem excluídos da pesquisa.

Em relação ao tempo de hospitalização, verificou-se que, no MMC, esse tempo foi menor. $\mathrm{O}$ referido tempo de permanência hospitalar constituise em um dos aspectos positivos do MMC, pois a estabilidade fisiológica, a eficiência da sucção e, conseqüentemente, o processo de alimentação, são efetivados mais rapidamente pela proximidade materna, favorecidos pelo contato pele a pele, o que comprova os benefícios do MMC, tornando imprescindível sua utilização nos países em desenvolvimento, pois o menor tempo de permanência hospitalar contribui para o restabelecimento da relação mãe-bebê, diminui o risco de infecção hospitalar, além de acarretar em menores custos para a saúde pública. ${ }^{15-21}$

O estado comportamental predominante nesta pesquisa foi o de alerta com inatividade, sendo esse processo considerado o ideal para a avaliação e intervenção, pois é o estado que mais favorece a interação, originando respostas com mais facilidade, além de ser imprescindível no desempenho da sucção. 6

Deve-se considerar que $50 \%$ dos bebês do Grupo 2 apresentavam uma postura extensora. Isso pode ser explicado pelo fato de o ambiente da UTIN ser hiper-estimulante e colaborar ainda mais para a desorganização da postura e do tônus do recémnascido pré-termo, o que dificulta todo o processo de sucção.4,5,7,22-24

Durante a reavaliação da sucção, foi verificado que houve dois aspectos com resultados estatisticamente significativos, relacionados com os estados comportamentais e com os sinais de estresse. Em relação aos estados comportamentais, foi verificado que os resultados encontrados no Grupo 2 podem ser explicados pelo ambiente desfavorável da UTIN, que juntamente com a imaturidade cerebral, os procedimentos invasivos e os manuseios excessivos, fazem com que esses bebês tenham dificuldades em passar de forma organizada pelos estados de consciência, dificultando o ritmo de sucção e a coordenação entre sucção, deglutição e respiração. Há então necessidade de estabelecer cuidados individualizados no intuito de viabilizar a estabilidade fisiológica e promover métodos alternativos de alimentação.22,24,25

Em relação aos sinais de estresse, verificaram-se quais sinais podem ser encontrados de acordo com cada subsistema. Nesse estudo, os sinais encontrados com maior freqüência foram: alterações respiratórias, soluço, variação do tônus, testa franzida e choro. 25,26

Justificam-se os inúmeros benefícios do MMC no que diz respeito à homeostase, prevenindo, portanto, o aparecimento dos sinais de estresse, pois o contato pele a pele favorece a estabilidade fisiológica e as pausas na respiração não aumentam com os procedimentos diários. É extremamente importante para a recuperação da fadiga, pois o ambiente do alojamento Mãe-canguru contém muitos estímulos que se assemelham ao ambiente intra-uterino. Traz, ainda, consideráveis benefícios para a ventilação como taxa de saturação de oxigênio aumentada, normalização da temperatura, além de contribuir para a regulação do sono e organização dos estados comportamentais favorecendo, dessa forma, o desempenho da sucção. 15,27-29

Quando comparada a avaliação da sucção com a reavaliação no Grupo 1, foi verificado que houve uma melhora estatisticamente significativa no ritmo de sucção. Os resultados satisfatórios no ritmo de sucção podem ser devidos à experiência da sucção através da estimulação não-nutritiva, além da aprendizagem das mães no processo de alimentação. No entanto, deve-se considerar o contexto fisiológico da alimentação, assim como a maturação neurológica para o pleno desenvolvimento da função, devendo-se considerar também outros fatores que podem influenciar esse processo, tais como: fome, freqüência cardíaca, saturação de oxigênio e estado comportamental.3,8,26,30 
Quando comparada a avaliação com a reavaliação no Grupo 2, o que se considerou estatisticamente significante foi em relação à coordenação entre sucção, deglutição e respiração. Acredita-se que a estimulação fonoaudiológica tenha sido a causa da mudança de comportamento ocorrida. Além do que, muitos autores afirmam que a idade gestacional não é o único fator determinante para o sucesso da alimentação por via oral, pois a partir de 32 semanas o recém-nascido prematuro tem condições de coordenar sucção e deglutição, com essa habilidade precedida de reflexos de tosse e vômito, desde que estimulado. Deve-se observar que a introdução precoce da alimentação por via oral pode antecipar todo o processo e, quando associada com a estimulação da sucção não-nutritiva, tem um importante papel na alimentação. No entanto, devem ser considerados também os sinais de aproximação e retraimento. $3,4,6,8,10,22,26,30$

Observou-se que todos os bebês foram favorecidos com a intervenção fonoaudiológica, pois com essa intervenção houve uma transição mais rápida da alimentação para a via oral, com melhor ganho de peso e, conseqüentemente, alta hospitalar precoce, o que contribuiu para o restabelecimento da relação mãe-bebê, favorecendo o processo da comunicação. Todavia os melhores resultados foram referentes ao Método Mãe-canguru, pois a proximidade materna facilitada pelo contato pele a pele contribuiu para o estabelecimento da amamentação em livre demanda, controle das habilidades fisiológicas, com melhor organização nos ciclos do sono, além de uma melhor organização dos estados comportamentais, havendo, portanto, menos sinais de estresse, quando comparado com o grupo controle.

Conclui-se, então, que o Método Mãe-Canguru constitui uma ótima alternativa para países em desenvolvimento, pois além de favorecer a relação mãe-bebê, facilitando a comunicação, contribui para a efetividade da alimentação, favorecendo o aleitamento materno, e conseqüentemente as funções de sugar e deglutir, além de diminuir o tempo de permanência hospitalar, acarretando menores custos para a saúde pública.

\section{Referências}

1. Charpak N, Calume ZF, Hamel A. O método mãe-canguru. Rio de Janeiro: McGraw Hill; 1999.

2. Charpak N, Calume ZF, Ruiz JG. The Bogotá Declaration on Kangaroo Mother Care: conclusions at the second internacional workshop on the method. Acta Paediatr 2000; 89: 1137-40.

3. Hernandez AM. Atuacão fonoaudiológica em neonatologia: uma proposta de intervenção. In: Andrade CRF. Fonoaudiologia em berçário normal e de risco. São Paulo: Lovise; 1996. p.43-98.

4. Silva RNM. Efeitos da sucção não-nutritiva. In: Lopes SMB, Lopes JMA. Folow-up do recém-nascido de alto risco. Rio de Janeiro: Medsi; 1999. p. 309-16.

5. Dargassies SA. Desarrollo neurologico del recien nascido de termino y premature. Buenos Aires: Panamericana; 1977.

6. Brazelton TB. As forças vitais do recém-nascido. In: Brazelton TB. O desenvolvimento do apego. Porto Alegre: Artes Médicas; 1988. p. 110-37.

7. Amiel-Tison C. Neurologic evaluation of the maturity of newborn infants. Arch Dis Child 1968; 43: 89-93.

8. Xavier C. Assistência à alimentação de bebês hospitalizados. In: Basseto MCA, Brock R, Wajnsztejn R. Neonatologia um convite à atuação fonoaudióloga. São Paulo: Lovise; 1998. p. 255-83.
9. Quintella T, Silva AA, Botelho MIMR. Distúrbios da deglutição e aspiração na infância. In: Furkin AM, Santini, CS. Disfagias orofaríngeas. Carapicuíba: Pró-fono; 1999. p. 61-96.

10. Neiva FCB. Análise evolutiva do padrão de sucção e a influência da estimulação através da sucção não-nutritiva em recém-nascidos prematuros [tese doutorado]. São Paulo: Faculdade de Medicina da Universidade de São Paulo; 2003.

11. Bernardis KC, Marchi SON. Sucção não-nutritiva de recém-nascidos a termo e pré-termo: um estudo descritivo e comparativo. Pró-fono 1998; 10: 8-15.

12. OMS (Organización Mundial de la Salud). Nuevas tendencias y metodos de asistencia maternoinfantil em los servicios de la salud. Ginebra; La Organización; 1976. 106p. [Série de Informes Técnicos, 600].

13. Callil VMLT. Caracterização do recém-nascido pré-termo. In: Leone CR, Trochin DMR. Assistência integrada ao recém-nascido. São Paulo: Atheneu; 1996. p. 22-32.

14. Pursley DWM, Cloherty JP. Identificando o recém-nascido de alto risco e avaliando a idade gestacional. In: Cloherty JP, Stark AR. Manual de neonatologia. Belo Horizonte: Medsi; 2000. p. 39-54.

15. Ludington-Hoe SM, Anderson GC, Simpson S, Hollingsead A, Argote LA, Rey H. Birth-related fatighe in 34-36-week preterm neonates: rapide recovery with very early kangaroo 
(skin-to-skin) care. J Obstet Gynecol Neonatal Nurs 1999; 28: 94-103.

16. Moran M, Radyminsky SG, Higgins KR, Dowling DA, Miller MJ, Anderson GC. Maternal kangaroo (skin-to-skin) care in the NICU beginning 4 hours postbirth. Med Cons Network 1999; 24: 74-9.

17. Lima G, Quintero-Romero S, Cattaneo A. Feasibility, acceptability and cost of kangaroo mother care in Recife, Brazil. Ann Trop Paediatr 2000; 20: 22-6.

18. Charpak N, Ruiz-Pelaez JG, Figueroa CZ, Charpak Y. A randomized controlled trial of kangaroo mother care: results of follow-up at one year of corrected age. Pediatrics 2001; 108: 1072-9.

19. Kirsten GF, Bergman NJ, Hann FM. Kangaroo mother care in the nursey. Pediatr Clin North Am 2001; 48: 443-52.

20. Ministério da Saúde. Secretaria de Políticas da Saúde. Área da Saúde da Criança. Atenção humanizada ao recémnascido de baixo peso: método Mãe-Canguru. Brasília (DF): Ministério da Saúde; 2002.

21. Conde-Agudelo A, Diaz-Rossello JL, Belizan JM. Kangaroo mother care to reduce morbidity and mortality in low birthweight infants. The Cochrane Library (Oxford) 2000 Disponível em: URL:http//www.bireme.br. [2002 Jun 28]

22. Glass P. O recém-nascido vulnerável e o ambiente da unidade de tratamento intensivo neonatal. In: Avery GB, Fletcher M, Donald M. Fisiopatologia e tratamento do recém-nascido. São Paulo: Medsi; 1999. p. 79-93.

23. Sheahan MS, Brockway NF, Teckin JS. A criança de alto risco. In: Teckin JS. Fisioterapia pediátrica. São Paulo: Artmed; 2002. p. 69-97.
24. Symington A, Pinelli J. Developmental care for promoting development an preventing morbidity in preterm infants. The Cochrane Library (Oxford) 2001. Disponível em URL:http://www.bireme.br [2002 Jun 28]

25. Als H. A synactive model of neonatal behavioral organization: framework for assessment of neurobehavioral development in the premature infant support of infant and parents in the neonatal intensive care environment. Phys Occup Ther Pediatr 1986; 6: 3-55.

26. Als H, Lawhon G, Duffy F, McAnulty GB, Gibes-Grossman $\mathrm{R}$, Blikman JG. Individualized developmental care for the very low-birth-weight preterm infant: medical and neurofunctional effects. JAMA 1994; 272: 853-8.

27. Withielaw A. Skin-to-skin contact in the care of very low birth weight babies. Mat Child Health 1986; 7: 242-6.

28. Wheller JL, Johnson M, Collie L, Sutherland D, Chapman C. Promoting breasfeeding in the neonatal intensive care unit. Breastfeed Rev 1999; 7: 15-8.

29. Feldman R, Weller A, Sirota L, Eidelman AL. Skin-to-skin contact (kangaroo care) promotes self-regulation in premature infants: sleep-wake cyclicity, arousal modulation, and sustained exploration. Dev Psychol 2002; 38: 194-207.

30. Bernabaum JC, Pereira GR, Watkins JB, Peckham GJ Nonnutritive sucking during feeding enhances growth and maturation in premature infants. Pediatrics 1983; 71: 41-5

Recebido em 25 de agosto de 2004

Versão final apresentada em 26 de outubro de 2004

Aprovado em 9 de novembro de 2004 